\title{
INERTIAL EFFECTS IN NON-EQUILIBRIUM THERMODYNAMICS
}

\author{
J.M. Rubí and A. Pérez-Madrid \\ Departament de Fisica Fonamental, \\ Facultat de Física \\ Universitat de Barcelona, \\ Diagonal 647, 08028 Barcelona, Spain
}

\begin{abstract}
We discuss inertial effects in systems outside equilibrium within the framework of non-equilibrium thermodynamics. By introducing a Gibbs equation in which the entropy depends on the probability density, we are able to describe a system of Brownian particles immersed in a heat bath in both inertial and diffusion regimes. In the former, a relaxation equation for the diffusion current is obtained whereas in the latter we recover Fick's law. Our approach, which uses the elements of the theory of internal degrees of freedom, constitutes the mesoscopic version of a previous analysis which takes into account the kinetic energy of diffusion.
\end{abstract}




\section{INTRODUCTION}

The macroscopic behavior of systems outside equilibrium is well described by non-equilibrium thermodynamics [1]. This theory, which is based upon the local equilibrium hypothesis, provides the phenomenological equations for the currents appearing in the conservation laws which are necessary to establish a complete set of differential equations accounting for the evolution of the fields. The success of the theory is a consequence of its simplicity, its solid statistical mechanical foundations, and of the great variety of situations to which it may be applied.

At the mesoscopic level, for times long enough, belonging to the diffusion regime, the dynamics of a system of particles immersed in a heat bath is governed by the Smoluchowski equation. From this equation, which involves the probability density for the positions of the particles, we can derive the diffusion equation accounting for the evolution of the density field. The diffusion equation may in turn be obtained from non-equilibrium thermodynamics by using the Fick's law in the balance of mass. If we want to describe the dynamics at shorter time scales, governed by the corresponding Fokker-Planck equation, the lack of equilibration in momentum space of the particles makes it necessary to incorporate inertial terms in the hydrodynamic description.

Our purpose in this paper is precisely to discuss how the scope of non-equilibiurm thermodynamics goes beyond the diffusion regime and may be extended to shorter time scales defining the inertial regime. In this context, it was already shown in Ref. 11] that phenomenological equations containing the derivatives of the diffusion currents, and consequently giving rise to relaxation equations for such currents, can be obtained from a proper definition of the internal energy. In this definition, the internal energy excludes the kinetic energies of all components with respect to the centre of mass, and is then directly related to the molecular nature of the system as it only contains contributions due to thermal agitation and molecular interactions. After a short time, the system enters the diffusion regime, in which the diffusion currents obey Fick's law. This is precisely the regime in which non-equilibrium thermodynamics has been usually applied.

Motivated by the fact that in the inertial regime the molecular nature of the system manifests itself, and with the aim of describing the process keeping in mind that nature, we will present in this paper a mesoscopic treatment of the process, complementary to the macroscopic analysis, which is based on the formulation of non-equilibrium thermodynamics in $\underline{\Gamma} \equiv(\vec{r}, \vec{u})$-space. In this way we will be able to obtain differential equations for the hydrodynamic fields which will describe the behavior of the system in both diffusion and inertial regimes. In the diffusion regime we will recover the phenomenological equation of non-equilibrium thermodynamics whereas in the inertial regime our formalism will give rise to a relaxation equation. Our derivation is inspired in the introduction of internal degrees of freedom [1], [2] to deal with the thermodynamical description of the system when different relaxation processes, occurring in well separated time scales, take place and constitute a more elaborated version of this theory which is put in its proper perspective.

The paper is organized in the following way: In section II we establish the local conservation laws which are derived from the continuity equation in $\underline{\Gamma-s p a c e . ~ T h e ~ p e r t i n e n t ~ f o r m u l a t i o n ~ o f ~ n o n-e q u i l i b r i u m ~ t h e r m o d y n a m i c s ~ i s ~ c a r r i e d ~ o u t ~}$ in section III, whereas in section IV we analyze the dynamics in both diffusion and inertial regimes. Finally, in the last section we summarize our main results and clarify the scope of non-equilibrium thermodynamics.

\section{CONSERVATION LAWS}

In the hydrodynamic regime, the evolution of systems outside equilibrium is described by a certain number of balance equations expressing the local conservation of the hydrodynamic fields. Since we are interested in the mesoscopic behavior of the system, our description will be carried out in terms of the distribution function of the particles.

To proceed, we will focus on an intentionally simple and illustrative example as is the case of a dilute suspension of Brownian particles that will be referred to as the Brownian 'gas', which was previously introduced in [3], [4] to study diffusion and thermal diffusion processes in the context of the theory of internal degrees of freedom. Quite generally, we will assume that, in the absence of external forces, the single particle distribution function $f(\vec{r}, \vec{u}, t)$ evolves according to the conservation law in phase space

$$
\frac{\partial f}{\partial t}=-\vec{u} \cdot \nabla f-\frac{\partial}{\partial \vec{u}} \cdot \vec{J}_{\vec{u}}
$$

which introduces the current $\vec{J}_{\vec{u}}$ that at this level constitutes an unspecified quantity.

The first moments of the distribution will be identified with the conserved fields. The density of particles $\rho_{B}$ can then be defined as

$$
\rho_{B}(\vec{r}, t)=m \int f(\vec{u}, \vec{r}, t) d \vec{u},
$$


where $m$ is the mass of a particle. In the same way the local momentum of the particles, $\rho_{B} \vec{v}_{B}$, is

$$
\rho_{B} \vec{v}_{B}(\vec{r}, t)=m \int f(\vec{u}, \vec{r}, t) \vec{u} d \vec{u},
$$

where $\vec{v}_{B}$ is the velocity field of the Brownian particles. Finally, the internal energy of the gas $u_{B}$ is defined as

$$
\rho_{B} u_{B}(\vec{r}, t)=\frac{m}{2} \int f\left(\vec{u}-\vec{v}_{B}\right)^{2} d \vec{u} .
$$

The balance equations for the hydrodynamic fields of the gas are the evolution equations for the moments that follow by using the conservation law (11). Then, if we differentiate eq. (2) with respect to time we obtain

$$
\frac{\partial \rho_{B}}{\partial t}=-\nabla \cdot \rho_{B} \vec{v}_{B}
$$

where we have used eq. (3) and the fact that $f$ goes to zero sufficiently rapidly for large $\vec{u}$. The balance equation for the momentum of the particles also follows from its definition (3). After differentiating this expression with respect to time and using again eq. (11) one obtains

$$
\frac{\partial}{\partial t} \rho_{B} \vec{v}_{B}=-m \int \vec{u} \vec{u} \cdot \nabla f d \vec{u}+m \int \vec{J}_{\vec{u}} d \vec{u}
$$

where we have also used the asymptotic behavior of $f$. Introducing now the pressure tensor for the particles

$$
\begin{gathered}
\overrightarrow{\vec{P}}_{B}=m \int f\left(\vec{u}-\vec{v}_{B}\right)\left(\vec{u}-\vec{v}_{B}\right) d \vec{u}= \\
m \int f \vec{u} \vec{u} d \vec{u}-\rho \vec{v}_{B} \vec{v}_{B}
\end{gathered}
$$

eq. (6) transforms into

$$
\rho_{B} \frac{d \vec{v}_{B}}{d t}=-\nabla \cdot \overrightarrow{\vec{P}}_{B}(\vec{r}, t)+m \int \vec{J}_{\vec{u}} d \vec{u}
$$

where we have defined the total derivative as

$$
\frac{d}{d t} \equiv \frac{\partial}{\partial t}+\vec{v}_{B} \cdot \nabla
$$

Finally, following the procedure previously indicated we can derive the internal energy balance equation from its definition (4). One obtains

$$
\rho_{B} \frac{d u_{B}}{d t}=-\nabla \cdot \vec{J}_{q}-\overrightarrow{\vec{P}}_{B}: \nabla \vec{v}_{B}+m \int \vec{J}_{\vec{u}} \cdot\left(\vec{u}-\vec{v}_{B}\right) d \vec{u},
$$

where we have defined the heat flow

$$
\vec{J}_{q}=\frac{1}{2} m \int f\left(\vec{u}-\vec{v}_{B}\right)^{2}\left(\vec{u}-\vec{v}_{B}\right) d \vec{u} .
$$

It is clear that the balance equations for the gas do not constitute, by themselves, a closed set of differential equations since the current $\vec{J}_{\vec{u}}$ has not been specified. The integrals involving such a current, appearing in eqs. (6) and (10), account for the interchange of momentum and internal energy between the Brownian particles and the heat bath, respectively. Its expression will be obtained from an appropriate formulation of non-equilibrium thermodynamics in $\underline{\Gamma}$-space, which will be carried out in the next section. 


\section{NON-EQUILIBRIUM THERMODYNAMICS}

In the diffusion regime, non-equilibrium thermodynamics establishes the Gibbs equation in which changes in the entropy are related to variations in the density of the particles. Since our purpose is to carry out a thermodynamical description of the system also in the inertial regime, in which the molecular individuality manifests, we will generalize

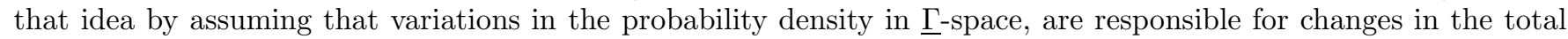
entropy of the system, $S$. Our starting point will then be to formulate the Gibbs equation

$$
\delta S=-\frac{m}{T} \int \mu(\underline{\Gamma}, t) \delta f(\underline{\Gamma}, t) d \underline{\Gamma}
$$

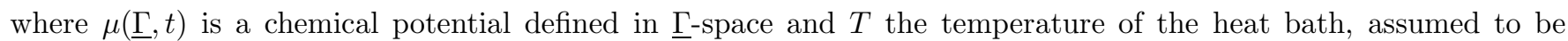
constant.

The expression of the chemical potential can be identified by requiring that the Gibbs equation (12) must be compatible with the Gibbs entropy postulate [t]. This postulate establishes that

$$
S=-k \int f \ln f / f^{l e q} d \underline{\Gamma}+S^{l . e q}
$$

where $f$ is an arbitrary distribution function and the local equilibrium distribution $f^{l . e q}$. is the local Maxwellian

$$
f^{l . e q \cdot}(\underline{\Gamma}, t)=\exp \left\{\left(\mu_{B}(\vec{r}, t)-\frac{1}{2} u^{2}\right) m / k T\right\}
$$

with $\mu_{B}(\vec{r}, t)$ being the chemical potential of the particles at local equilibrium. Moreover, $S^{l . e .}$ is the total entropy at local equilibrium whose variations are given by the Gibbs equation

$$
\delta S^{\text {l.e. }}=-\frac{1}{T} \int \mu_{B}(\vec{r}, t) \delta \rho_{B}(\vec{r}, t) d \vec{r}
$$

By differentiating eq. (13) and comparing with eq. (12) we obtain, after using eq. (15), the expression of the chemical potential $\mu(\vec{u}, \vec{r}, t)$ that is written

$$
\mu(\vec{u}, \vec{r}, t)=\mu_{B}+\frac{k T}{m} \ln \left(f / f^{l . e q .}\right)
$$

where $k$ is the Boltzmann constant. The Gibbs equation then resembles its corresponding expression for a mixture in which the different species would correspond to the different values of $\underline{\Gamma}$, which may then be interpreted as an internal coordinate or degree of freedom [1]|.

Taking the time derivative in (12) and using (11) and (16) we obtain the entropy balance equation

$$
\frac{d S}{d t}=-\int \overrightarrow{J_{s}} \cdot d \vec{s}+\int \sigma d \vec{r}
$$

where the entropy flux is given by

$$
\overrightarrow{J_{s}}=-k \int f(\ln f-1) \vec{u} d \vec{u}
$$

and the entropy production reads

$$
\sigma=-k \int \vec{J}_{\vec{u}} \cdot \frac{\partial}{\partial \vec{u}} \ln \left(f / f^{l . e q \cdot}\right) d \vec{u}
$$

This quantity is subjected to the restriction $\sigma \geq 0$, which constitutes the formulation of the second principle. The linear laws corresponding to eq. (19) can be formulated by assuming isotropy and locality in $\vec{u}$-space. One has

$$
\vec{J}_{\vec{u}}=-k L_{\vec{u} \vec{u}} \frac{\partial}{\partial \vec{u}} \ln \left(f / f^{l . e q \cdot}\right)
$$

where $L_{\vec{u} \vec{u}}$ is a phenomenological coefficient which may in general depend on the state variables. This expression can alternatively be written as 


$$
\vec{J}_{\vec{u}}=-\beta\left(f \vec{u}+\frac{k T}{m} \frac{\partial f}{\partial \vec{u}}\right)
$$

where $\beta$ is a coefficient defined as

$$
\beta \equiv m L_{\vec{u} \vec{u}} / f T
$$

which in first approximation is assumed constant. Equation (21) can then be used in (1) giving

$$
\frac{\partial f}{\partial t}=-\vec{u} \cdot \nabla f+\beta \frac{\partial}{\partial \vec{u}} \cdot\left(f \vec{u}+\frac{k T}{m} \frac{\partial f}{\partial \vec{u}}\right),
$$

This expression constitutes the Fokker-Planck equation accounting for the evolution of the distribution function of the gas.

\section{INERTIAL AND DIFFUSION REGIMES}

Having obtained the expression for the current $\vec{J}_{\vec{u}}$ from non-equilibrium thermodynamics, we will proceed first to formulate the complete set of hydrodynamic equations for the gas of Brownian particles. Using eq. (21) in (8) and performing the corresponding integral we then obtain

$$
\rho_{B} \frac{d \vec{v}_{B}}{d t}+\nabla \cdot \overrightarrow{\vec{P}}_{B}(\vec{r}, t)=-\beta \rho_{B} \vec{v}_{B}
$$

In the internal energy balance equation (10), the integral involving the current $\vec{J}_{\vec{u}}$ can also be computed by using $(21)$ arriving at

$$
\rho_{B} \frac{d u_{B}}{d t}=-\nabla \cdot \vec{J}_{q}-\overrightarrow{\vec{P}}_{B}: \nabla \vec{v}_{B}-2 \beta \rho_{B}\left(u_{B}-u_{B}^{e q \cdot}\right)
$$

where $u_{B}^{e q .}=\frac{3}{2} \frac{k T}{m}$.

It becomes clear from eq. (24) that the coefficient $\beta^{-1}$ introduces a characteristic time scale in our system. The case $t \lesssim \beta^{-1}$ corresponds with the inertial regime. To discuss this regime our starting point will be eq. (24) that by using eq. (5) and the definition (9) can be expressed in terms of the diffusion current $\vec{J}_{D} \equiv \rho_{B} \vec{v}_{B}$ in the form

$$
\vec{J}_{D}=-\frac{\beta^{-1}}{1+\beta^{-1} \nabla \cdot \vec{v}_{B}}\left\{\nabla \cdot \overrightarrow{\vec{P}}_{B}+\frac{d \vec{J}_{D}}{d t}\right\} .
$$

This expression constitutes a relaxation equation for the diffusion current. Up to first order in $\beta^{-1}$, it yields

$$
\vec{J}_{D}=-\beta^{-1}\left\{\nabla \cdot \overrightarrow{\vec{P}}_{B}+\frac{d \vec{J}_{D}}{d t}\right\}
$$

In order to compute the pressure tensor we need to know the form of the distribution function. We will consider the case discussed in ref. [1] in which the initial distribution is given by

$$
f(\underline{\Gamma}, 0)=\exp \left\{\left(\mu_{B}(\vec{r}, 0)-\frac{1}{2}\left(\vec{u}-\vec{v}_{B}(\vec{r}, 0)\right)^{2}\right) m / k T_{B}\right\} .
$$

The solution of the Fokker-Planck equation at later times has also the same Gaussian form and is given by

$$
f(\underline{\Gamma}, t)=\exp \left\{\left(\mu_{B}(\vec{r}, t)-\frac{1}{2}\left(\vec{u}-\vec{v}_{B}(\vec{r}, t)\right)^{2}\right) m / k T_{B}\right\},
$$

provided the conditions $\nabla_{i} v_{B, i}=\frac{1}{3} \nabla \cdot \vec{v}_{B} \delta_{i j}$ and $\nabla T_{B}=0$ be fulfilled. Using eq. (29) in the definition of the pressure tensor given through eq. (7) we obtain

$$
\overrightarrow{\vec{P}}_{B}=p_{B} \overrightarrow{\vec{U}}=\frac{k T_{B}}{m} \rho_{B} \overrightarrow{\vec{U}}
$$


where $\overrightarrow{\vec{U}}$ is the unit tensor. Therefore, eq. (27) gives

$$
\vec{J}_{D}=-\beta^{-1}\left(\frac{k T_{B}}{m} \nabla \rho_{B}+\frac{d \vec{J}_{D}}{d t}\right)
$$

Likewise, when the distribution fuunction is given by eq. (29) one has $\vec{J}_{q}=0$, and $u_{B}=\frac{3}{2} \frac{k T_{B}}{m}$, which enables us to obtain the relaxation equation of the temperature $T_{B}$ from eq.(25)

$$
\frac{d T_{B}}{d t}=-\frac{2}{3} T_{B} \nabla \cdot \vec{v}_{B}-2 \beta\left(T_{B}-T\right) .
$$

Moreover, eq. (24) transforms into

$$
\rho_{B} \frac{d \vec{v}_{B}}{d t}=-\frac{k T_{B}}{m} \nabla \rho_{B}-\beta \rho_{B} \vec{v}_{B} .
$$

Equations (5), (32), and (33), with the equation of state (30), results in a closed set of differential equations describing the evolution of the hydrodynamic fields, $\rho_{B}, \vec{v}_{B}, T_{B}$, and $p_{B}$.

If in addition, we assume that $T_{B}=T$, eq. (31) becomes

$$
\vec{J}_{D}=-D \nabla \rho_{B}-\beta^{-1} \frac{d \vec{J}_{D}}{d t}
$$

with

$$
D=\frac{k T}{m \beta}
$$

being the diffusion coefficient.

Inserting the relaxation equation (34) in the balance of mass (5) one then obtains

$$
\begin{aligned}
& \frac{\partial \rho_{B}}{\partial t}=D \nabla^{2} \rho_{B}-D \beta^{-1} \nabla \cdot \frac{\partial \nabla \rho_{B}}{\partial t} \\
& =D \nabla^{2} \rho_{B}-D^{2} \beta^{-1} \nabla^{2} \nabla^{2} \rho_{B}
\end{aligned}
$$

where we have neglected quadratic terms in the gradients. The corresponding equation in $k$-space gives [6]

$$
\frac{\partial \rho_{B}}{\partial t}=-k^{2} D\left(1+D \beta^{-1} k^{2}\right) \rho_{B} .
$$

These equations contains corrections of higher order in the gradient which appear beyond the first Chapman-Enskog approximation. The term proportional to $k^{4}$ in eq. (37) corresponds to the Burnett approximation.

When we use the distribution function (29), with $T_{B}=T$, in the expression of the entropy production given in eq. (19), we obtain

$$
\begin{array}{r}
\sigma=-\frac{1}{T} \vec{J}_{D} \cdot\left\{\nabla \mu_{B}+\frac{d}{d t} \vec{v}_{B}\right\}= \\
-\frac{1}{T} \vec{J}_{D} \cdot\left\{\nabla\left(\mu_{B}+\frac{1}{2} \vec{v}_{B}^{2}\right)+\frac{\partial}{\partial t} \vec{v}_{B}\right\},
\end{array}
$$

where we have employed eq. (11) and the definition of the total derivative (9). This result agrees with the diffusive part of the entropy production for a mixture,

$$
\sigma=-\frac{1}{T} \sum_{k} \vec{J}_{k} \cdot\left\{\nabla\left(\mu_{k}^{*}+\frac{1}{2}\left(\vec{v}_{k}-\vec{v}\right)^{2}\right)+\frac{D}{D t}\left(\vec{v}_{k}-\vec{v}\right)\right\},
$$

which was obtained in ref. [1] from a standard Gibbs equation in which the internal energy does not include the kinetic energy of diffusion of the components. Eq. (39) corresponds with the case in which no external forces are 
present and $T=$ const. Here, $\vec{J}_{k}$ is the diffusion current of the $\mathrm{k}$-th component, $\mu_{k}^{*}$ is the chemical potential of the k-th component, which does not include the kinetic energy of diffusion, $\vec{v}=\rho^{-1} \sum_{k} \rho_{k} \vec{v}_{k}$ is the baricentric velocity, $\rho_{k}$, and $\vec{v}_{k}$ are the density and velocity of the k-th component, respectively, and $\rho$ is the total density.Moreover, the total derivative is taken with respect to the baricentric motion

$$
\frac{D}{D t}=\frac{\partial}{\partial t}+\vec{v} \cdot \nabla
$$

To arrive at eq. (38) from eq. (39) we have to consider that the mixture consists of two components: heat bath and Brownian particles, with $\rho_{B} \ll \rho_{H}$ and $\vec{v}_{H} \simeq 0$, resulting from the fact that the Brownian gas is very dilute. Here, $\rho_{H}$ and $\vec{v}_{H}$ are the density and velocity of the heat bath, respectively. These conditions imply that the baricentric velocity is a negligible quantity, and consequently that $D / D t \simeq \partial / \partial t$. Additionally, we have to identify the chemical potential of the particles in eq. (39) with our $\mu_{B}$.

For $t \gg \beta^{-1}$ one achieves the diffusion regime. Here, the inertial term can be neglected and eq. (33) becomes

$$
0=-\frac{k_{B} T}{m} \nabla \rho_{B}-\beta \rho_{B} \vec{v}_{B}
$$

which transforms into

$$
\vec{J}_{D}=-D \nabla \rho_{B}
$$

This expression corresponds to Fick's law. In this regime, the continuity equation (5) gives the Smoluchowski equation

$$
\frac{\partial \rho_{B}}{\partial t}=D \nabla^{2} \rho_{B}
$$

which describes the evolution of the density in the diffusion regime. This equation coincides with the diffusion equation since we have not considered interactions among particles.

To give an estimate of the characteristic time $\beta^{-1}$, let us consider the particular situation in which the particles are spheres and the problem is stationary. The friction coefficient per unit of mass is then given by the Stokes law [5]

$$
\beta=\frac{6 \pi \eta a}{m} .
$$

Substituting $m=(4 / 3) \pi a^{3} \rho_{p}$, with $a$ being the radius of the particle and $\rho_{p}$ its density, in this expression it gives

$$
\beta=\frac{9}{2} \frac{\eta}{a^{2} \rho_{p}}=\frac{9}{2} \frac{\nu}{a^{2}} \frac{\rho_{f l}}{\rho_{p}}
$$

where $\rho_{f l}$ is the density of the heat bath. This equation provides the time scale in which inertial effects should be considered. If the fluid is water for which $\nu \sim 10^{-2} \mathrm{~cm}^{3} / \mathrm{s}$ one has

$$
\beta^{-1} \sim 10^{2} a^{2} \frac{\rho_{p}}{\rho_{f l}}
$$

Assuming that the densities are not very different and $a \sim 10^{-5} \mathrm{~cm}$, a typical particle size, one has $\beta^{-1} \sim 10^{-8} \mathrm{~s}$. The smallness of this time then shows why non-equilibrium thermodynamics has been so successful when analyzing transport phenomena at sufficiently long times.

\section{DISCUSSION}

In this paper we have discussed the range of applications of non-equilibrium thermodynamics. In its more common formulation presented in [1], which has been successfully used to describe a wide variety of situations, this theory implicitly assumes that linear laws relating fluxes and forces are valid in the diffusion regime. In spite of this apparent restriction, it is worth emphasizing that the behavior of the system in the inertial regime, which precedes the diffusion regime, can also be described within the framework of the theory. As pointed out in the monography [1], when passing from an individualized description of the components, in which each of them has its proper velocity, to a global description characterized dynamically by the baricentric velocity, we are neglecting the kinetic energies of 
all components with respect to the center of mass, referred to as kinetic energy of diffusion. The consideration of this energy, which as commented in Ref. [i] is perhaps more accurate than the one corresponding to the baricentric velocity since it only contains contributions due to thermal agitation and molecular interactions of the particles, has implications in the definition of the internal energy, since one simply introduces it by subtraction from the total energy, kinetic and potential energies. A Gibbs equation can then be proposed in which the entropy is as usual a function of the internal energy, obtained by subtraction from the total energy the kinetic and potential energies of all the components, the specific volume and the mass fractions. The resulting entropy production contains inertial terms which give rise to relaxation equations.

In addition to the macroscopic treatment outlined previously and with the aim of making the analysis more in depth, we have presented in this paper a mesoscopic version of the process based upon non-equilibrium thermodynamics of systems with internal degrees of freedom. Maintaining the essentials of non-equilibrium thermodynamics, we have proposed a Gibbs equation where the entropy this time instead of depending on the density it depends on the

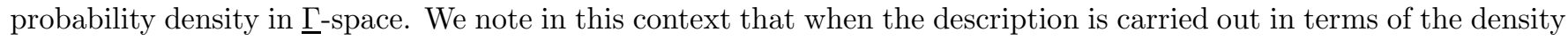
of the particles or of the probability density for the positions of the particles (since they respectively evolve according to the diffusion equation and the Smoluchowski equation) we lose information at times smaller than the characteristic time at which the system enters the diffusion regime. In this way we have derived the relaxation equation obtained from the macroscopic theory which enables one to analyze the inertial and diffusion regimes. In the inertial regime, the resulting differential equation for the density of Brownian particles contain corrections of higher order in the density gradient proper of the Burnett approximation.

The Gibbs equation we have proposed through eq. (12) is perfectly compatible with the entropy formulated through the Gibbs postulate, by an adequate choice of the chemical potential 田. Apart from this equivalence presented in section III , the Gibbs postulate, along with the assumption that the dynamics is governed by a Fokker-Planck equation, leads to the same entropy production, and consequently to the linear laws we have obtained. These features provide our Gibbs equation with solid foundations.

The mesoscopic approach presented here together with the macroscopic treatments addressed in refs. [1] and [7] then raise the question about the validity of formulating entropies including dissipative currents as independent variables. Such a possibility, which only may occurs when there exists a clear separation between the different time scales [4, is equivalent to formulate a standard Gibbs equation in which the internal energy takes into account the individual nature of the different components. We have corroborated this fact by means of a mesoscopic approach in terms of the single particle distribution function.

To end this paper it is worth pointing out that although our analysis has been presented in the simple case of a dilute gas of Brownian particles it could systematically be applied to more complex situations, thus arriving at a complete description of the systems, in both inertial and diffusion regimes, by using the method of non-equilibrium thermodynamics.

\section{ACKNOWLEDGMENTS}

We wish to thank Prof. P. Mazur for useful discussion. This work has been supported by DGICYT of the Spanish Government under grant PB95-0881.

[1] S. R. de Groot and P. Mazur, "Non-Equilibrium Thermodynamics", (Dover Publishing Co., New York, 1984). See in particular ch. IX, $\S 8$ and $\mathrm{X}, \S 6$.

[2] I. Prigogine and P. Mazur, Physica XIX, 241 (1953).

[3] A. Pérez-Madrid, J. M. Rubí and P. Mazur, Physica A, 212, 231, (1994).

[4] J.M. Rubí and P. Mazur, Physica A 250, (1998) 253.

[5] L.D. Landau, and E.M. Lifshitz, "Fluid Mechanics" (2nd edn), (Pergamon, Oxford, 1987).

[6] P.M.V. Résibois, M. de Leener. Classical Kinetic Theory of Fluids. (Wiley, New York, 1977). Ch. XII, sec. 6.3.

[7] K. Miyazaki, K. Kitahara and D. Bedeaux, Physica A 230, (1996) 600. 